\title{
Crystal structure of 18-oxabicyclo[8.6.3]nonadeca-2,9,17-trione, $\left(\mathrm{CHCOC}_{6} \mathrm{H}_{12} \mathrm{COC}_{2} \mathrm{H}_{3} \mathrm{OOC}\right)\left(\mathrm{C}_{6} \mathrm{H}_{12}\right)$
}

\author{
E.-M. Peters*.I, K. Peters', B. Seyberlich" and W. Tochtermann" \\ I Max-Planck-Institut für Festkörperforschung. Heisenbergstraße 1. D-70506 Stuttgart. Germany \\ II Institut für Organische Chemie der Universität Kiel. Olshausenstraße 40. D-24098 Kiel. Germany
}

Received November 29, 2000. CCDC-No. $1267 / 570$
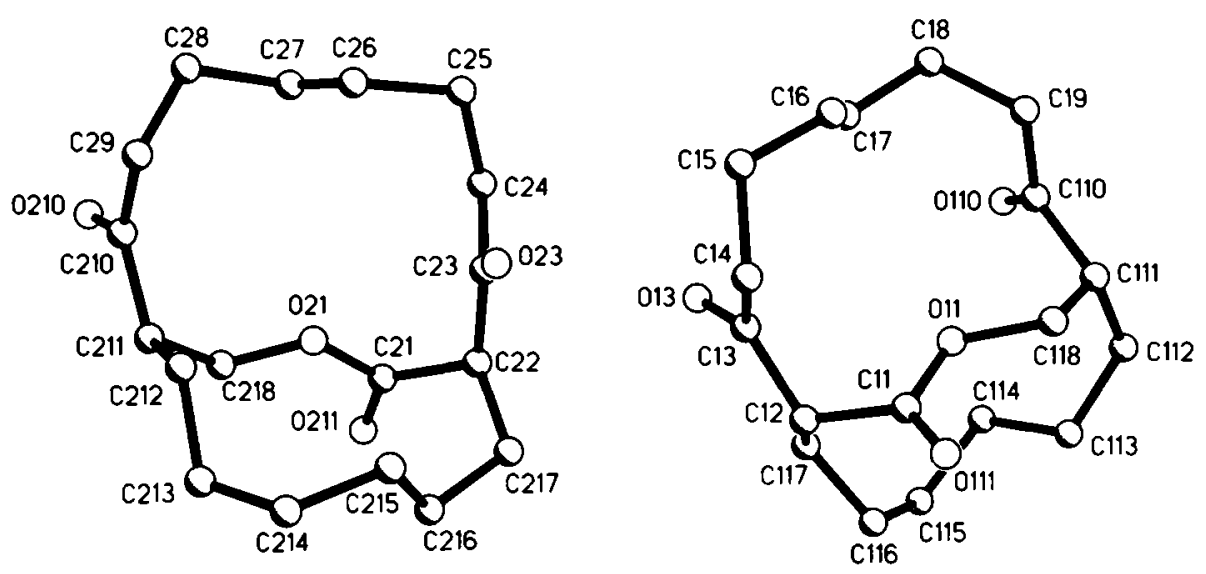

\section{Abstract}

$\mathrm{C}_{18} \mathrm{H}_{28} \mathrm{O}_{4}$, monoclinic, $P 12_{1} / c 1$ (No. 14), $a=17.360(2) \AA$,

$b=18.487(2) \AA, c=10.836(1) \AA, \beta=100.51(1)^{\circ}, V=3419.3 \AA^{3}$, $Z=8, R_{\mathrm{gt}}(F)=0.098, w R_{\text {ref }}\left(F^{2}\right)=0.340, T=293 \mathrm{~K}$.

\section{Source of material}

The title compound was obtained by ruthenium tetraoxide oxidation of a tetrahydrooxepin $[1,2]$.

Table 1. Data collection and handling.

\begin{tabular}{ll}
\hline Crystal: & colourless lump, size $0.4 \times 0.55 \times 0.6 \mathrm{~mm}$ \\
Wavelength: & Mo $K_{\alpha}$ radiation $(0.71073 \AA)$ \\
$\mu:$ & $0.83 \mathrm{~cm}^{-1}$ \\
Diffractometer, scan mode: & Bruker AXS P4, $\omega$ \\
$2 \theta_{\max }:$ & $55^{\circ}$ \\
$N(h k l)_{\text {measured, }} N(h k l)_{\text {unique: }}:$ & 8256,7841 \\
Criterion for $I_{\text {obs }}, N(h k l)_{\text {gt: }}:$ & $I_{\text {obs }}>2 \sigma\left(I_{\text {obs }}\right), 4521$ \\
$N(\text { param })_{\text {refined: }}$ & 397 \\
Program: & SHELXL-97 [3] \\
\hline
\end{tabular}

Table 2. Atomic coordinates and displacement parameters (in $\AA^{2}$ ).

\begin{tabular}{|c|c|c|c|c|c|}
\hline Atom & Site & $x$ & $y$ & $\Sigma$ & $U_{\text {iso }}$ \\
\hline$H(12)$ & $4 e$ & 0.4854 & 1.0257 & 0.8681 & 0.08 \\
\hline$H(14 A)$ & $4 e$ & 0.4992 & 1.1577 & 1.0112 & 0.08 \\
\hline$H(14 B)$ & $4 e$ & 0.4082 & 1.1519 & 0.9709 & 0.08 \\
\hline$H(15 A)$ & $4 e$ & 0.4120 & 1.2656 & 0.8662 & 0.08 \\
\hline$H(15 B)$ & $4 e$ & 0.4288 & 1.2689 & 1.0129 & 0.08 \\
\hline$H(16 A)$ & $4 e$ & 0.5660 & 1.2679 & 0.9971 & 0.08 \\
\hline$H(16 B)$ & $4 e$ & 0.5200 & 1.3403 & 0.9675 & 0.08 \\
\hline $\mathrm{H}(17 \mathrm{~A})$ & $4 e$ & 0.5604 & 1.2471 & 0.7845 & 0.08 \\
\hline$H(17 B)$ & $4 e$ & 0.5103 & 1.3171 & 0.7514 & 0.08 \\
\hline$H(18 A)$ & $4 e$ & 0.6209 & 1.3846 & 0.8544 & 0.08 \\
\hline $\mathrm{H}(18 \mathrm{~B})$ & $4 e$ & 0.6304 & 1.3490 & 0.7269 & 0.08 \\
\hline$H(19 A)$ & $4 e$ & 0.7426 & 1.3348 & 0.8909 & 0.08 \\
\hline $\mathrm{H}(19 \mathrm{~B})$ & $4 e$ & 0.6920 & 1.2845 & 0.9607 & 0.08 \\
\hline$H(111)$ & $4 e$ & 0.8051 & 1.1966 & 0.9424 & 0.08 \\
\hline$H(12 A)$ & $4 e$ & 0.8170 & 1.1435 & 0.7380 & 0.08 \\
\hline$H(12 B)$ & $4 e$ & 0.8530 & 1.1056 & 0.8639 & 0.08 \\
\hline$H(13 A)$ & $4 e$ & 0.8002 & 1.0146 & 0.7470 & 0.08 \\
\hline$H(13 B)$ & $4 e$ & 0.7376 & 1.0299 & 0.8310 & 0.08 \\
\hline$H(14 A)$ & $4 e$ & 0.7254 & 1.0672 & 0.5775 & 0.08 \\
\hline $\mathrm{H}(14 \mathrm{~B})$ & $4 e$ & 0.6681 & 1.1000 & 0.6591 & 0.08 \\
\hline $\mathrm{H}(15 \mathrm{~A})$ & $4 e$ & 0.6154 & 0.9954 & 0.5366 & 0.08 \\
\hline$H(15 B)$ & $4 e$ & 0.6788 & 0.9480 & 0.6212 & 0.08 \\
\hline$H(16 A)$ & $4 e$ & 0.6212 & 0.9753 & 0.7960 & 0.08 \\
\hline$H(16 B)$ & $4 e$ & 0.5634 & 0.9336 & 0.6921 & 0.08 \\
\hline$H(17 A)$ & $4 e$ & 0.5486 & 1.0819 & 0.6667 & 0.08 \\
\hline $\mathrm{H}(17 \mathrm{~B})$ & $4 e$ & 0.4814 & 1.0246 & 0.6575 & 0.08 \\
\hline$H(18 A)$ & $4 e$ & 0.7337 & 1.0958 & 1.0026 & 0.08 \\
\hline $\mathrm{H}(18 \mathrm{~B})$ & $4 e$ & 0.7034 & 1.1731 & 1.0317 & 0.08 \\
\hline
\end{tabular}


Table 2. Continued.

\begin{tabular}{|c|c|c|c|c|c|}
\hline$A(0) 1$ & Site & $x$ & $y$ & $z$ & $U_{i w 1}$ \\
\hline $\mathrm{H}(22 \mathrm{~A})$ & te & $0.286 ?$ & 1.1074 & 0.5760 & 0.08 \\
\hline $\mathrm{H}(2+A)$ & te & 0.3029 & 1.2479 & 0.5974 & 0.08 \\
\hline $\mathrm{H}(2+\mathrm{B})$ & te & 0.21 .38 & 1.2362 & $0.5+44$ & 0.08 \\
\hline $\mathrm{H}(25 \mathrm{~A})$ & 40 & $0.2+11$ & 1.3518 & 0.6264 & 0.08 \\
\hline $\mathrm{H}(25 \mathrm{~B})$ & te & 0.2696 & 1.3179 & 0.7594 & 0.08 \\
\hline$H(26 A)$ & to & 0.1 .361 & 1.2724 & 0.7565 & 0.08 \\
\hline $\mathrm{H}(26 \mathrm{~B})$ & te & 0.1392 & 1.3570 & 0.7574 & 0.08 \\
\hline $\mathrm{H}(27 \mathrm{~A})$ & te & 0.0915 & 1.2722 & 0.5595 & 0.08 \\
\hline$H(27 B)$ & te & 0.0981 & 1.3573 & 0.5558 & 0.08 \\
\hline $\mathrm{H}(28 \mathrm{~A})$ & te & 0.0185 & 1.3568 & 0.7306 & 0.08 \\
\hline $\mathrm{H}(28 \mathrm{~B})$ & te & -0.0286 & 1.3454 & 0.5950 & 0.08 \\
\hline $\mathrm{H}(29 \mathrm{~A})$ & te & 0.0097 & 1.2371 & 0.7734 & 0.08 \\
\hline$H(29 B)$ & te & -0.0738 & 1.2704 & 0.7308 & 0.08 \\
\hline $\mathrm{H}(211)$ & te & -0.0681 & 1.0939 & 0.6089 & 0.08 \\
\hline
\end{tabular}

Table 2. Continued.

\begin{tabular}{|c|c|c|c|c|c|}
\hline Atom & Site & $x$ & $y$ & $=$ & $U_{1 \times 1}$ \\
\hline $\mathrm{H}(12 \mathrm{C})$ & $4 e^{\prime}$ & -0.0246 & 1.1184 & 0.8214 & 0.08 \\
\hline$H(I 2 D)$ & te & 0.0608 & 1.1294 & 0.7966 & 0.08 \\
\hline $\mathrm{H}(\mathrm{I} 3 \mathrm{C})$ & te & -0.0178 & 0.9993 & 0.8120 & 0.08 \\
\hline$H(\mid 3 D)$ & te & 0.0378 & 1.0005 & 0.7135 & 0.08 \\
\hline $\mathrm{H}(\mathrm{I}+\mathrm{C})$ & te & 0.1012 & 0.9530 & 0.9045 & 0.08 \\
\hline$H(\mid 4 D)$ & te & 0.0878 & 1.0256 & 0.9718 & 0.08 \\
\hline $\mathrm{H}(15 \mathrm{C})$ & te & 0.1706 & 1.0848 & 0.8520 & 0.08 \\
\hline$H(15 D)$ & te & 0.2146 & 1.0276 & 0.9454 & 0.08 \\
\hline $\mathrm{H}(\mathrm{I} 6 \mathrm{C})$ & te & 0.1643 & 0.9917 & 0.6922 & 0.08 \\
\hline$H(16 D)$ & $4 e$ & 0.2263 & 0.9500 & 0.7892 & 0.08 \\
\hline $\mathrm{H}(17 \mathrm{C})$ & $4 e$ & 0.3108 & 1.0538 & 0.7880 & 0.08 \\
\hline$H(17 D)$ & $4 e$ & 0.2995 & 1.0045 & 0.6688 & 0.08 \\
\hline $\mathrm{H}(18 \mathrm{C})$ & $4 e$ & 0.0302 & 1.0493 & 0.5311 & 0.08 \\
\hline H(18D) & $4 e$ & 0.0174 & 1.1252 & 0.4651 & 0.08 \\
\hline
\end{tabular}

Table 3. Atomic coordinates and displacement parameters (in $\AA^{2}$ ).

\begin{tabular}{|c|c|c|c|c|c|c|c|c|c|c|}
\hline Atom & Site & $x$ & $y$ & $z$ & $U_{11}$ & $U_{22}$ & $U_{33}$ & $U_{12}$ & $U_{13}$ & $U_{23}$ \\
\hline$O(11)$ & te & $0.6353(1)$ & $1.1246(1)$ & $0.8862(2)$ & $0.043(1)$ & $0.057(1)$ & $0.039(1)$ & $-0.0086(9)$ & $0.0037(8)$ & $0.0070(9)$ \\
\hline$C(11)$ & $4 e$ & $0.5899(2)$ & $1.0755(2)$ & $0.9287(3)$ & $0.052(2)$ & $0.060(2)$ & $0.047(2)$ & $-0.012(1)$ & $0.012(1)$ & $0.008(1)$ \\
\hline$C(12)$ & 4e & $0.5150(2)$ & $1.0642(2)$ & $0.8357(3)$ & $0.057(2)$ & $0.085(3)$ & $0.061(2)$ & $-0.028(2)$ & $0.009(2)$ & $0.008(2)$ \\
\hline$C(13)$ & $4 e$ & $0.4654(2)$ & $1.1337(3)$ & $0.8271(3)$ & $0.041(2)$ & $0.130(4)$ & $0.055(2)$ & $-0.010(2)$ & $0.011(2)$ & $0.011(2)$ \\
\hline$O(13)$ & $4 e$ & $0.4325(2)$ & $1.1558(2)$ & $0.7251(3)$ & $0.062(2)$ & $0.151(3)$ & $0.061(2)$ & $0.009(2)$ & $0.004(1)$ & $0.008(2)$ \\
\hline$C(14)$ & $4 e$ & $0.4553(3)$ & $1.1707(3)$ & $0.9460(4)$ & $0.060(2)$ & $0.150(5)$ & $0.064(2)$ & $0.014(3)$ & $0.021(2)$ & $0.000(3)$ \\
\hline$C(15)$ & $4 e$ & $0.4494(3)$ & $1.2523(4)$ & $0.9405(4)$ & $0.065(3)$ & $0.172(6)$ & $0.067(3)$ & $0.034(3)$ & $0.018(2)$ & $-0.011(3)$ \\
\hline$C(16)$ & $4 e$ & $0.5251(3)$ & $1.2914(3)$ & $0.9377(4)$ & $0.092(3)$ & $0.116(4)$ & $0.058(2)$ & $0.026(3)$ & $0.010(2)$ & $-0.015(2)$ \\
\hline$C(17)$ & $4 e$ & $0.5521(3)$ & $1.2958(2)$ & $0.8124(3)$ & $0.090(3)$ & $0.082(3)$ & $0.050(2)$ & $0.028(2)$ & $0.004(2)$ & $-0.004(2)$ \\
\hline$C(18)$ & $4 e$ & $0.6255(3)$ & $1.3388(2)$ & $0.8129(4)$ & $0.123(4)$ & $0.060(2)$ & $0.058(2)$ & $0.012(2)$ & $0.015(2)$ & $0.003(2)$ \\
\hline$C(19)$ & $4 e$ & $0.6997(3)$ & $1.3004(2)$ & $0.8785(3)$ & $0.093(3)$ & $0.056(2)$ & $0.051(2)$ & $-0.015(2)$ & $0.007(2)$ & $0.001(2)$ \\
\hline$C(110)$ & $4 e$ & $0.7223(2)$ & $1.2360(2)$ & $0.8072(3)$ & $0.067(2)$ & $0.059(2)$ & $0.043(2)$ & $-0.018(2)$ & $0.013(1)$ & $0.000(1)$ \\
\hline$O(110)$ & $4 e$ & $0.7118(2)$ & $1.2357(2)$ & $0.6945(2)$ & $0.145(3)$ & $0.075(2)$ & $0.043(1)$ & $0.002(2)$ & $0.025(2)$ & $0.007(1)$ \\
\hline$C(111)$ & $4 e$ & $0.7626(2)$ & $1.1740(2)$ & $0.8830(3)$ & $0.041(2)$ & $0.068(2)$ & $0.052(2)$ & $-0.011(1)$ & $0.006(1)$ & $-0.000(2)$ \\
\hline$C(113)$ & $4 e$ & $0.7618(3)$ & $1.0495(2)$ & $0.7644(4)$ & $0.084(3)$ & $0.080(3)$ & $0.075(3)$ & $0.028(2)$ & $0.019(2)$ & $0.007(2)$ \\
\hline$C(114)$ & $4 e$ & $0.7001(3)$ & $1.0582(3)$ & $0.6488(4)$ & $0.103(3)$ & $0.090(3)$ & $0.057(2)$ & $0.021(3)$ & $0.029(2)$ & $0.005(2)$ \\
\hline$C(115)$ & $4 e$ & $0.6461(4)$ & $0.9906(3)$ & $0.6205(5)$ & $0.126(4)$ & $0.076(3)$ & $0.076(3)$ & $0.007(3)$ & $0.011(3)$ & $-0.024(2)$ \\
\hline$C(116)$ & $4 e$ & $0.5909(3)$ & $0.9790(2)$ & $0.7117(5)$ & $0.128(4)$ & $0.062(2)$ & $0.087(3)$ & $-0.021(3)$ & $0.010(3)$ & $-0.014(2)$ \\
\hline$C(117)$ & $4 e$ & $0.5305(3)$ & $1.0404(3)$ & $0.7081(4)$ & $0.075(3)$ & $0.097(3)$ & $0.064(2)$ & $-0.024(2)$ & $-0.005(2)$ & $-0008(2)$ \\
\hline$C(118)$ & $4 e$ & $0.7103(2)$ & $1.1401(2)$ & $0.9650(3)$ & $0.048(2)$ & $0.058(2)$ & $0.040(1)$ & $-0.007(1)$ & $0.002(1)$ & $0.005(1)$ \\
\hline$O(21)$ & $4 e$ & $0.1110(1)$ & $1.1208(1)$ & $0.5984(2)$ & $0.059(1)$ & $0.056(1)$ & $0.056(1)$ & $0.005(1)$ & $0.009(1)$ & $-0.011(1)$ \\
\hline$C(21)$ & $4 e$ & $0.1686(3)$ & $1.0909(3)$ & $0.5493(4)$ & $0.108(3)$ & $0.099(3)$ & $0.047(2)$ & $0.051(3)$ & $0.009(2)$ & $-0.011(2)$ \\
\hline$C(22)$ & $4 e$ & $0.2485(3)$ & $1.1040(3)$ & $0.6330(4)$ & $0.072(2)$ & $0.114(4)$ & $0.064(2)$ & $0.036(2)$ & $0.025(2)$ & $0.014(2)$ \\
\hline $\mathrm{C}(23)$ & $4 e$ & $0.2525(2)$ & $1.1759(3)$ & $0.7007(4)$ & $0.051(2)$ & $0.095(3)$ & $0.076(3)$ & $0.005(2)$ & $0.018(2)$ & $0.012(2)$ \\
\hline$O(23)$ & $4 e$ & $0.2597(2)$ & $1.1799(2)$ & $0.8130(3)$ & $0.078(2)$ & $0.098(2)$ & $0.070(2)$ & $-0.009(2)$ & $0.009(1)$ & $0.004(2)$ \\
\hline$C(24)$ & $4 e$ & $0.2519(3)$ & $1.2430(4)$ & $0.6210(6)$ & $0.083(3)$ & $0.144(5)$ & $0.109(4)$ & $-0.001(3)$ & $0.027(3)$ & $0.048(4)$ \\
\hline$C(25)$ & $4 e$ & $0.2334(5)$ & $1.3117(4)$ & $0.6806(8)$ & $0.186(8)$ & $0.076(4)$ & $0.148(7)$ & $-0.025(4)$ & $-0.018(6)$ & $0.022(4)$ \\
\hline$C(26)$ & $4 e$ & $0.1446(8)$ & $1.3145(6)$ & $0.7072(9)$ & $0.39(2)$ & $0.159(8)$ & $0.133(7)$ & $0.15(1)$ & $-0.03(1)$ & $-0.046(6)$ \\
\hline $\mathrm{C}(27)$ & $4 e$ & $0.0909(7)$ & $1.3162(4)$ & $0.6082(8)$ & $0.26(1)$ & $0.092(5)$ & $0.126(6)$ & $0.006(6)$ & $-0.011(7)$ & $0.015(4)$ \\
\hline$C(28)$ & $4 e$ & $0.0097(6)$ & $1.3233(4)$ & $0.6605(9)$ & $0.198(9)$ & $0.092(5)$ & $0.176(8)$ & $0.056(5)$ & $0.012(7)$ & $-0.012(5)$ \\
\hline $\mathrm{O}(210)$ & $4 e$ & $-0.0785(3)$ & $1.2170(3)$ & $0.5039(4)$ & $0.111(3)$ & $0.159(4)$ & $0.114(3)$ & $0.054(3)$ & $-0.018(2)$ & $0.022(3)$ \\
\hline$C(211)$ & $4 e$ & $-0.0203(2)$ & $1.1226(3)$ & $0.6332(4)$ & $0.052(2)$ & $0.092(3)$ & $0.081(3)$ & $-0.010(2)$ & $-0.001(2)$ & $0.007(2)$ \\
\hline$O(211)$ & $4 e$ & $0.1515(3)$ & $1.0473(3)$ & $0.4621(4)$ & $0.159(4)$ & $0.149(4)$ & $0.098(3)$ & $0.040(3)$ & $0.019(3)$ & $-0.038(3)$ \\
\hline$C(212)$ & $4 e$ & $0.0124(2)$ & $1.1029(3)$ & $0.7695(4)$ & $0.056(2)$ & $0.093(3)$ & $0.081(3)$ & $-0.002(2)$ & $0.016(2)$ & $0.017(2)$ \\
\hline$C(213)$ & $4 e$ & $0.0286(3)$ & $1.0219(3)$ & $0.7912(5)$ & $0.094(3)$ & $0.083(3)$ & $0.115(4)$ & $-0.038(3)$ & $0.009(3)$ & $0.021(3)$ \\
\hline$C(214)$ & $4 e$ & $0.0975(3)$ & $1.0050(3)$ & $0.8937(5)$ & $0.124(4)$ & $0.067(3)$ & $0.084(3)$ & $-0.015(3)$ & $0.010(3)$ & $0.024(2)$ \\
\hline$C(215)$ & $4 e$ & $0.1760(3)$ & $1.0334(2)$ & $0.8691(4)$ & $0.091(3)$ & $0.056(2)$ & $0.066(2)$ & $0.009(2)$ & $-0.003(2)$ & $0.012(2)$ \\
\hline$C(216)$ & $4 e$ & $0.2074(3)$ & $0.9978(2)$ & $0.7622(4)$ & $0.121(4)$ & $0.058(2)$ & $0.075(3)$ & $0.032(2)$ & $-0.013(3)$ & $-0.001(2)$ \\
\hline$C(217)$ & $4 e$ & $0.2730(3)$ & $1.0382(3)$ & $0.7156(5)$ & $0.093(3)$ & $0.104(4)$ & $0.080(3)$ & $0.050(3)$ & $0.014(2)$ & $0.007(3)$ \\
\hline$C(218)$ & $4 e$ & $0.0333(3)$ & $1.1011(2)$ & $0.5452(4)$ & $0.091(3)$ & $0.062(2)$ & $0.067(2)$ & $-0.004(2)$ & $-0.006(2)$ & $-0.006(2)$ \\
\hline
\end{tabular}


Acknowledgment. The finacial support by the Deutsche Forschungsgemeinschaft is gratefully acknowledged.

\section{References}

1. Seyberlich, B.: Tochtermann W.: Synthese bicyclischer Makrolide - ein Beitrag zu Struktur-Geruchsbeziehungen, unpublished results.

2. Tochtermann, W.: Dibbern, R.; Haase, M.: Bruhn, T.: Wolff, C.: Die Rutheniumtetroxid-Oxidation von 3,6-Alkano-4,5-oligomethylenoxepinen. Ein neuer Weg zu makrocyclischen Di-, Tri- und Tetraketonen. Chem. Ber. 124 (1991) 923-931.

3. Sheldrick, G. M.: SHELXL-97, a program for refining crystal structures, University of Göttingen, Germany 1997. 